\title{
Light-matter interactions in high quality manganese- doped two-dimensional molybdenum diselenide
}

\author{
Sheng $\mathrm{Liu}^{1 \dagger}$, Yaze $\mathrm{Wu}^{2,6 \dagger}$, Xue Liu ${ }^{1}$, Andres Granados del Aguila ${ }^{1}$, Fengyuan Xuan ${ }^{2}$, \\ Apoorva Chaturvedi ${ }^{3}$, Hua Zhang ${ }^{4,5}$, Su Ying Quek ${ }^{2,6^{*}}$ and Qihua Xiong ${ }^{1,7^{*}}$
}

\begin{abstract}
Introducing magnetic dopants into twodimensional transition metal dichalcogenides has recently attracted considerable attention due to its promising applications in spintronics and valleytronics. Herein we realized manganese-doped molybdenum diselenide $\left(\mathrm{MoSe}_{2}\right)$ single crystal via chemical vapor transport (CVT) reaction, containing up to $2.9 \%$ (atomic concentration) Mn dopants, and investigated the light-matter interaction in these samples. We observed a suppressed trion intensity, a longer photoluminescence lifetime, and prominent blue- and red-shift of $\mathrm{E}_{2 \mathrm{~g}}^{2}$ (in-plane) and $\boldsymbol{A}_{1 \mathrm{~g}}$ (out-of-plane) Raman modes, respectively. Moreover, the Mn dopants increase the valley Zeeman splitting of the $\mathrm{MoSe}_{2}$ monolayer by $\sim 50 \%$, while preserving the linear dependence on magnetic field. First-principles calculations indicate that the spin-polarized deep level defect states are formed due to the Mn substitutional dopants in the $\mathrm{MoSe}_{2}$ lattice. The resulting defect potential favors the funnelling of excitons towards the defects. The Mn dopants reduce the magnitude of the interatomic force constants, explaining the red-shift of the $\mathrm{A}_{1 \mathrm{~g}}$ mode. The $\mathrm{Mn}$ atoms and their immediate Mo and Se neighbors carry significant magnetic moments, which enhance the observed exciton $\boldsymbol{g}$-factors due to the exchange interactions affecting defect-bound excitons.
\end{abstract}

Keywords: first-principles calculations, exciton funnelling, twodimensional semiconductor, magnetic doping, valley-Zeeman effect

\section{INTRODUCTION}

Over the past decade, two-dimensional (2D) semiconductors, especially the group VIB H-phase transition metal dichalcogenides (TMDs) with chemical formula $\mathrm{MX}_{2}(\mathrm{M}=\mathrm{Mo}$ and $\mathrm{W} ; \mathrm{X}=\mathrm{S}$ and $\mathrm{Se}$ ), have been intensively investigated. These monolayer TMDs possess remarkable physical properties, including an indirect-to-direct band gap transition from few-layer to monolayer [1,2], room temperature excitons with binding energy up to $\sim 200 \mathrm{meV}$ [3-6], valley polarization [7-10], and valley Hall effect $[11,12]$. In order to fully realize the potential of TMDs, preparation of TMD monolayers with high crystal quality is of great necessity. Chemical vapor deposition (CVD) and chemical vapor transport (CVT) reaction are two of the most widely used methods to synthesize TMDs. The CVD method produces on-substrate monoto fewlayer nanosheets. However, due to the fast and nonequilibrium deposition, those CVD monolayers often contain great amounts of defects including vacancies, grain boundaries, dislocations and residual strain [1316]. On the other hand, CVT inside the sealed ampules provides large single crystals of TMDs with high quality, profiting from the long-time growth under mild and equilibrium reactions [17]. The mechanically exfoliated monolayers from those as-grown TMD crystals have shown perfect optical and optoelectronic performances in previous research $[2,3,7,11,18-20]$.

For intrinsic semiconductors, alloying and chemical

\footnotetext{
${ }^{1}$ Division of Physics and Applied Physics, School of Physical and Mathematical Sciences, Nanyang Technological University, Singapore 637371, Singapore

${ }^{2}$ Department of Physics, National University of Singapore, 2 Science Drive 3, Singapore 117551, Singapore

${ }^{3}$ School of Materials Science and Engineering, Nanyang Technological University, Singapore 639798, Singapore

${ }^{4}$ Department of Chemistry, City University of Hong Kong, Hong Kong, China

${ }^{5}$ Hong Kong Branch of National Precious Metals Material Engineering Research Center (NPMM), City University of Hong Kong, Hong Kong, China

${ }^{6}$ Centre for Advanced 2D Materials, National University of Singapore, Singapore 117546, Singapore

${ }^{7}$ State Key Laboratory of Low-Dimensional Quantum Physics and Department of Physics, Tsinghua University, Beijing 100084, China

${ }^{\dagger}$ These two authors contributed equally to this paper.

* Corresponding authors (emails: Qihua@ntu.edu.sg or qihua_xiong@tsinghua.edu.cn (Xiong Q); phyqsy@nus.edu.sg (Quek SY))
} 
doping are indispensable ways to enhance their properties and applications. By analogy, chemical doping will also open new opportunities for those 2D TMDs. By substituting the cation or anion of the binary TMDs partially with heteroatoms during the CVD or CVT growth, ternary alloy or chemically doped TMDs such as $\mathrm{Mo}_{x} \mathrm{~W}_{1-x} \mathrm{X}_{2}$ and $\mathrm{MS}_{2 x} \mathrm{Se}_{2(1-x)}$ can be obtained [10,21,22]. Within the same chemical family (i.e., Mo, W, S, and Se), alloying provides fully tuneable chemical compositions $(0<x<1)[10,23,24]$. Those ternary alloys will bestow a large band gap tunability $(\sim 0.6 \mathrm{eV})$ to the TMDs and thus extend the wavelength range towards relevant optoelectronic devices. Those component elements have identical valence electronic structure, facilitating the alloying among themselves. However, it also prevents the alloyed TMDs from forming novel electronic band structure and gaining excess carriers. To vary the intrinsic properties of TMDs out of the box, dopants with unlike valence electronic structure need to be brought into the crystal lattice of TMDs.

The transition metal elements neighboring to the Group VIB column in the periodic table are potential candidates for unlike-element doping of TMDs. Previous CVD studies have had difficulties in the growth of Nband $\mathrm{Mn}$-doped $\mathrm{MoS}_{2}$ monolayers when grown on $\mathrm{SiO}_{2} / \mathrm{Si}$ substrates, and degraded optical qualities were observed on those doped monolayers $[25,26]$. The high formation energy of such systems makes it more difficult to grow high-quality monolayers when there is a lattice mismatch between the substrate and the monolayer, and van der Waals ( $\mathrm{vdW}$ ) epitaxial growth on a dangling-bond-free surface can potentially relax the lattice mismatch and improve the quality of the as-grown system $[25,27,28]$. To date, the growth outcomes for such unlike-element doping of TMDs have not been satisfactory. Herein, we report a well-designed CVT synthesis of Mn-doped $\mathrm{MoSe}_{2}$ single crystals under a thermodynamically controlled environment, using $\mathrm{AlCl}_{3}$ as a transport agent. Profiting from the layer-by-layer vdW growth within a single $\mathrm{Mn}$-doped $\mathrm{MoSe}_{2}$ single crystal with no substrate, the as-grown $\mathrm{MoSe}_{2}$ contains $\mathrm{Mn}$ dopants up to $2.9 \%$ (atomic concentration), showing equivalent crystal quality as compared with the pristine $\mathrm{MoSe}_{2}$ under X-ray diffraction (XRD) and scanning transmission electron microscopy (STEM) investigations. Light-matter interactions in these high-quality samples were investigated using photoluminescence (PL), time-resolved photoluminescence (TRPL) and Raman spectroscopies. These measurements reveal that the Mn dopants enhance the PL lifetimes and suppress the trion peak, and the $A_{1 g}$ Raman mode red-shifts, compared with the pristine $\mathrm{MoSe}_{2}$. Our density functional theory (DFT) calculations show that the Mn dopants result in deep level defect states and suggest that excitons will be funnelled toward the dopants. We also predict that the magnitude of the interatomic force constants will be reduced, leading to a redshift in the $A_{1 g}$ mode as observed in experiment. Although long-range order and ferromagnetism are not realized, our measurements reveal a $\sim 50 \%$ enhancement in the exciton $g$-factor compared with the pristine $\mathrm{MoSe}_{2}$. This enhancement is consistent with the local net magnetic moments predicted for the Mn dopants and surrounding atoms and arises from strong exchange interactions affecting defect-bound excitons. Our results pave the way for investigating the effect of doping of unlike-elements to 2D TMDs and highlight the potential significance of Mn-doped $\mathrm{MoSe}_{2}$ in spintronics and valleytronics applications.

\section{MATERIALS AND METHODS}

\section{Sample preparation}

The powder source of molybdenum (Aldrich, mean particle size $<5 \mu \mathrm{m}$, purity $99.9 \%$ ), manganese (Aldrich, mean particle size $<45 \mu \mathrm{m}$, purity $99.5 \%$ ) and selenium (Aldrich, mean particle size $<250 \mu \mathrm{m}$, purity $99.95 \%$ ) were loaded in a vacuum-sealed quartz tube, in stoichiometric proportions $\left(\mathrm{Mn}_{(0.05-0.1)} \mathrm{Mo}_{(0.95-0.9)} \mathrm{Se}_{2}\right)$. A small amount of $\mathrm{AlCl}_{3}$ was also loaded as a transport agent. The $\mathrm{AlCl}_{3}$ can create more Mn-enriched atmosphere during the reaction than other common transport agents. All powder sources were placed in one side of the sealed tube, and the tube was then put into a tube furnace, aligning to the middle of two heating zones which can be set to different temperatures. In the first week, the source and empty ends of the tube were set at 800 and $900^{\circ} \mathrm{C}$, respectively, for pre-reaction and gasifying the source adequately. In this reversed temperature gradient, no deposition of solid would take place. In the following two weeks, the source and empty ends of the tube were set at 1000 and $900^{\circ} \mathrm{C}$, respectively, the temperature gradient drove the transport and reaction of chemical vapors, making nucleation and crystal growth happen at the low temperature end. After two weeks, millimeter-sized crystals with flake appearance can be obtained. The pristine $\mathrm{MoSe}_{2}$ crystal was purchased from HQ Graphene. The as-grown $\mathrm{Mn}$-doped $\mathrm{MoSe}_{2}$ and pristine crystals were exfoliated using the scotch tape method, and the mono- and few-layers were transferred onto $\mathrm{Si} / \mathrm{SiO}_{2}$ $(285 \mathrm{~nm})$ substrates. 


\section{STEM}

The Mn-doped $\mathrm{MoSe}_{2}$ samples were transferred onto a copper grid with lacy carbon film for STEM measurement. STEM images were taken on a JEOL $2100 \mathrm{~F}$ with a STEM detector, which was operated at $120 \mathrm{kV}$.

\section{Raman scattering measurements}

Raman scattering measurements were performed in back scattering geometry using a micro-Raman spectrometer (Horiba JY T64000). The excitation laser (532 nm solid laser) was introduced using a $100 \times$ lens, and Raman signals were collected through the same lens. A forestage triple grating system with following $1800 \mathrm{l} / \mathrm{cm}$ grating was used to disperse the Raman signal to achieve a frequency as low as $6 \mathrm{~cm}^{-1}$.

\section{Photoluminescence measurements}

Photoluminescence spectra were taken in back scattering geometry using a micro-Raman spectrometer (Horiba HR Evolution). A laser $(633 \mathrm{~nm}, \mathrm{He}-\mathrm{Ne})$ was introduced using a $50 \times$ long focus lens, and emission signals were collected through the same lens, resolved by a $300 \mathrm{l} / \mathrm{cm}$ grating and finally went to a liquid nitrogen cooled CCD detector. A 633-nm notch filter was equipped to block the excitation line. For measurement of PL mapping, the excitation laser was modulated under DuoScan mode, which is a unique hardware module to scan the laser beam on a given area using software-controlled mirrors. For temperature-dependent PL, the sample was mounted in a cryostat with circulating liquid nitrogen or helium. Laser excitation and signal were going through a quartz window. For magnetic-field-dependent PL, the field was generated by a superconductor foil with circulating direct current (DC).

\section{Time-resolved photoluminescence measurements}

The fluorescence lifetime measurement was performed using a confocal microscopy with a $50 \times$ long focus lens. A pulse excitation ( $400 \mathrm{~nm}, 8 \mathrm{MHz}, 100 \mathrm{fs}$ ) was introduced to the sample, and the PL emission from the sample was analyzed by a single photon avalanche diode (SPAD) to resolve the time decay. A long-pass (458 $\mathrm{nm}$ ) and 800-nm short-pass filters were used to select out the PL.

\section{First-principles calculations}

Spin-polarized DFT calculations were performed on supercell configurations using the plane-wave Vienna $A b$ initio Simulation Package (VASP) code [29]. The projector augmented wave (PAW) approach [30,31] with the local density approximation (LDA) to the exchange cor- relation functional [32] was used. A Hubbard $U$ term [33] ( $U=3$ and $4 \mathrm{eV}[34,35]$ ) was added to account for the strong correlation effects in Mn. The results for $U=3$ and $4 \mathrm{eV}$ were qualitatively similar. Given the ferromagnetic alignment of the Mn dopants in previous studies [36,37], our supercells contained one $\mathrm{Mn}$ per cell. $4 \times 4 \times 1$ and $6 \times 6 \times 1$ cells were used in this study. The plane wave energy cutoff was $450 \mathrm{eV}$ and the Brillouin zone was sampled by Monkhorst-Pack $k$ point mesh [38], including the $\Gamma$ point with a density equivalent to $24 \times 24 \times 1$ per unit cell of $\mathrm{MoSe}_{2}$. The results were unchanged when the $k$-mesh density or plane-wave cutoff was increased. For phonon calculations, we used a vacuum height of $11 \AA$ and for the other calculations, a vacuum height of $17 \AA$ was used. The atomic coordinates were fully relaxed using the conjugate gradient algorithm and the electron density was optimized using the blocked Davidson iteration scheme until the maximum energy difference between iterations was less than $10^{-9} \mathrm{eV}$ and the residual force was less than $0.003 \mathrm{eV}^{-1}$. Phonon calculations were performed using the density functional perturbation theory (DFPT) in VASP and force constants were extracted using Phonopy [39]. Spatial charge distribution of the systems was performed using Bader Charge analysis [40] with a fine FFT mesh of $980 \times 980 \times 1200$, at which convergence with respect to fine FFT mesh density was reached. The charged defect formation energies were calculated using Corrections for Formation Energy and Eigenvalues for Charged Defect Simulations Package [41] with a $6 \times 6 \times 1$ supercell.

\section{RESULTS AND DISCUSSION}

A long-time CVT reaction in a sealed quartz tube was performed to obtain Mn-doped $\mathrm{MoSe}_{2}$ crystals, which is schematically illustrated in Fig. 1a. The precursors included molybdenum, manganese, and selenium powders in stoichiometric proportions $\left(\mathrm{Mn}_{(0.05-0.1)} \mathrm{Mo}_{(0.95-0.9)} \mathrm{Se}_{2}\right)$. A small amount of $\mathrm{AlCl}_{3}$ was loaded as a transport agent to create a Mn-enriched gaseous environment during the crystal growth [42]. After two weeks of growth, at the low temperature end of the tube, millimeter-sized crystals with flake appearance can be obtained (shown in Fig. 1b). The XRD pattern of the Mn-doped $\mathrm{MoSe}_{2}$ matches well with the pristine $\mathrm{MoSe}_{2}$ and the standard reference (Fig. S1), and the sharp peak at $2 \theta \sim 13.6^{\circ}$, which can be assigned to $\left(\begin{array}{lll}0 & 0 & 2\end{array}\right)$ crystal plane, demonstrates that the $\mathrm{Mn}$-doped $\mathrm{MoSe}_{2}$ has identical interlayer distance to the pristine $\mathrm{MoSe}_{2}$. The as-grown crystals are easy to exfoliate using scotch tape, like other $2 \mathrm{D}$ vdW crystals.

We then transferred the exfoliated thin layers to transmission electron microscope (TEM) grid for char- 
a

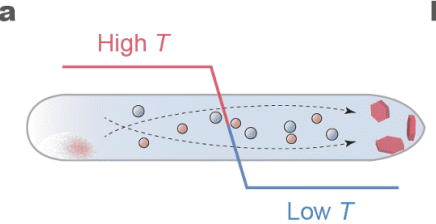

C

d
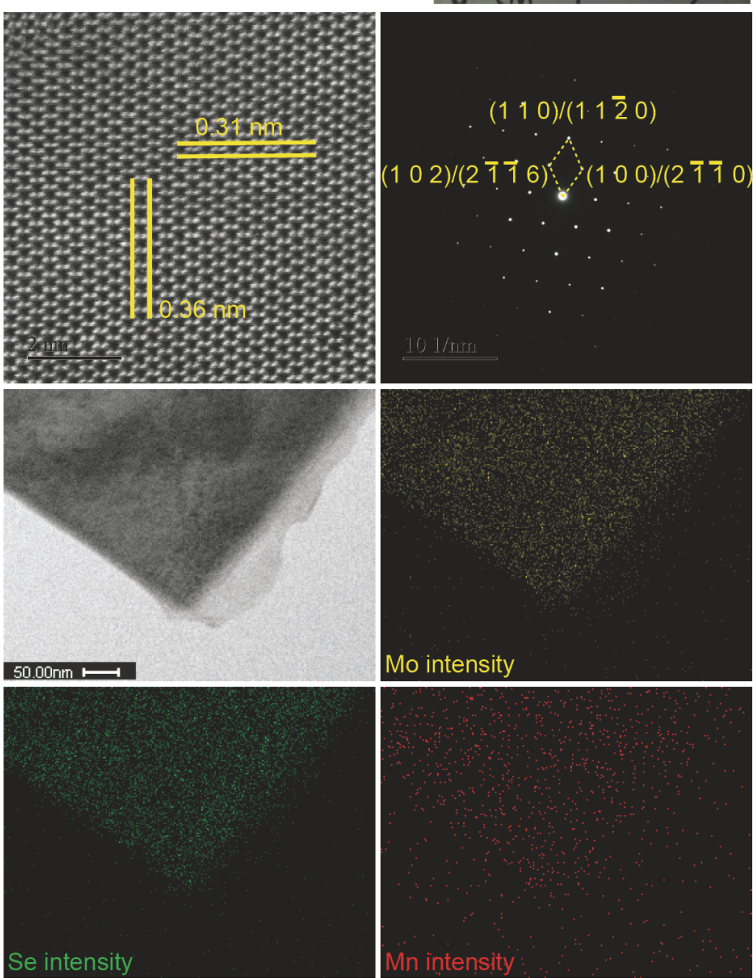

Figure 1 Synthesis and characterization of the pristine and Mn-doped $\mathrm{MoSe}_{2}$ crystals. (a) Schematic of the chemical vapor transport reaction in a sealed quartz tube; the chemical sources are loaded in the high temperature end and the products will nucleate and grow in the low temperature end. (b) Appearance and typical size of the grown Mn-doped $\mathrm{MoSe}_{2}$ single crystals. (c) HAADF-STEM image of an exfoliated fewlayer Mn-doped $\mathrm{MoSe}_{2}$ sample. (d) EDX intensity mapping of the Mndoped $\mathrm{MoSe}_{2}$ crystal. The top left panel is the TEM image of the region of interest on the sample, while the rest are the EDX intensity maps of all the elements of the Mn-doped $\mathrm{MoSe}_{2}$ crystal.

acterization. Fig. 1c shows the high-angle annular darkfield STEM (HAADF-STEM) image and the corresponding diffraction pattern, which both show clear hexagonal shape and indicate that the atoms are arranged in hexagonal lattice structure. The diameters of the hexatomic ring along zigzag and armchair directions are 0.31 and $0.36 \mathrm{~nm}$, respectively, which are in excellent agreement with previous experimental and theoretical results. The diffraction pattern is bright and sharp without any distortion or multi-fold, and the spots match the Miller indices of the main crystal planes (labelled with yellow numbers). Energy dispersive X-ray (EDX) in- tensity mapping was taken on the sample during the STEM measurement. The region of interest on the sample, with the EDX intensity mapping of Mo, Se and Mn is shown in Fig. 1d. This EDX intensity map shows that Mn is distributed across the entire region homogeneously. The characteristic X-rays of Mo and Se show very strong intensity, and the ratio of Mo to Se calculated from the signal intensity is approximately 1:2. The characteristic signal of $\mathrm{Mn}$ is much weaker than that of Mo and Se, showing about $2.9 \%$ of the total cations. However, the Mn intensity is still relatively pronounced as compared with the background noises, and the homogeneously distributed signal counts profile the sample appearance accordingly. These facts suggest that the as-grown crystal samples have a high-quality hexagonal lattice, which is consistent with the pristine $\mathrm{MoSe}_{2}$, while $\mathrm{Mn}$ atoms have been successfully doped during the crystal growth.

Mono- to few-layer samples on $\mathrm{Si} / \mathrm{SiO}_{2}(285 \mathrm{~nm})$ substrates were prepared by mechanical exfoliation using the pristine and $\mathrm{Mn}$-doped $\mathrm{MoSe}_{2}$ crystals. We measured the layer-dependent Raman spectra of both the pristine and Mn-doped $\mathrm{MoSe}_{2}$. Fig. 2a, b show the Raman spectra in the ranges of $A_{1 g}$ intralayer and $E_{2 g}^{2}$ interlayer shear modes, respectively. For each thickness, the $\mathrm{A}_{1 \mathrm{~g}}$ peak of the $\mathrm{Mn}$-doped $\mathrm{MoSe}_{2}$ red-shifts $\sim 1.8 \mathrm{~cm}^{-1}$ as compared with its pristine counterpart. On the contrary, the interlayer shear mode of the doped $\mathrm{MoSe}_{2}$ slightly blue-shifts $\sim 0.7 \mathrm{~cm}^{-1}$. Fig. $2 \mathrm{c}$ displays the layer dependent peak positions of $\mathrm{A}_{1 \mathrm{~g}}$ and $\mathrm{E}_{2 \mathrm{~g}}^{2}$ modes from all the samples. The Mn-doped and pristine $\mathrm{MoSe}_{2}$ show similar layerdependent trends of Raman peak positions, suggesting that the doping and induced structure changes are homogeneous within each layer. It is a reasonable result since the crystals are grown in a sealed tube under thermodynamic equilibrium environment. In addition, the STEM and EDX images exhibit no phase segregation or clustering of $\mathrm{Mn}$ atoms. The shifted phonon modes in the $\mathrm{Mn}$-doped $\mathrm{MoSe}_{2}$ compared with the pristine counterpart suggest that the dopants are substituted into the lattice rather than being adsorbed on the surface. The substitutional doping is further confirmed by first principles calculations of the Raman spectra, as discussed below.

It has been shown previously that substitutional transition metal dopants in TMDs can result in changes in the PL spectra [25,43]. To further explore the effects of Mn doping on the optical properties of $\mathrm{MoSe}_{2}$, we measured the PL spectra on the doped and pristine monolayers. Fig. 3a shows the PL mapping of two exfoliated samples (pristine and doped, respectively) at room temperature, 

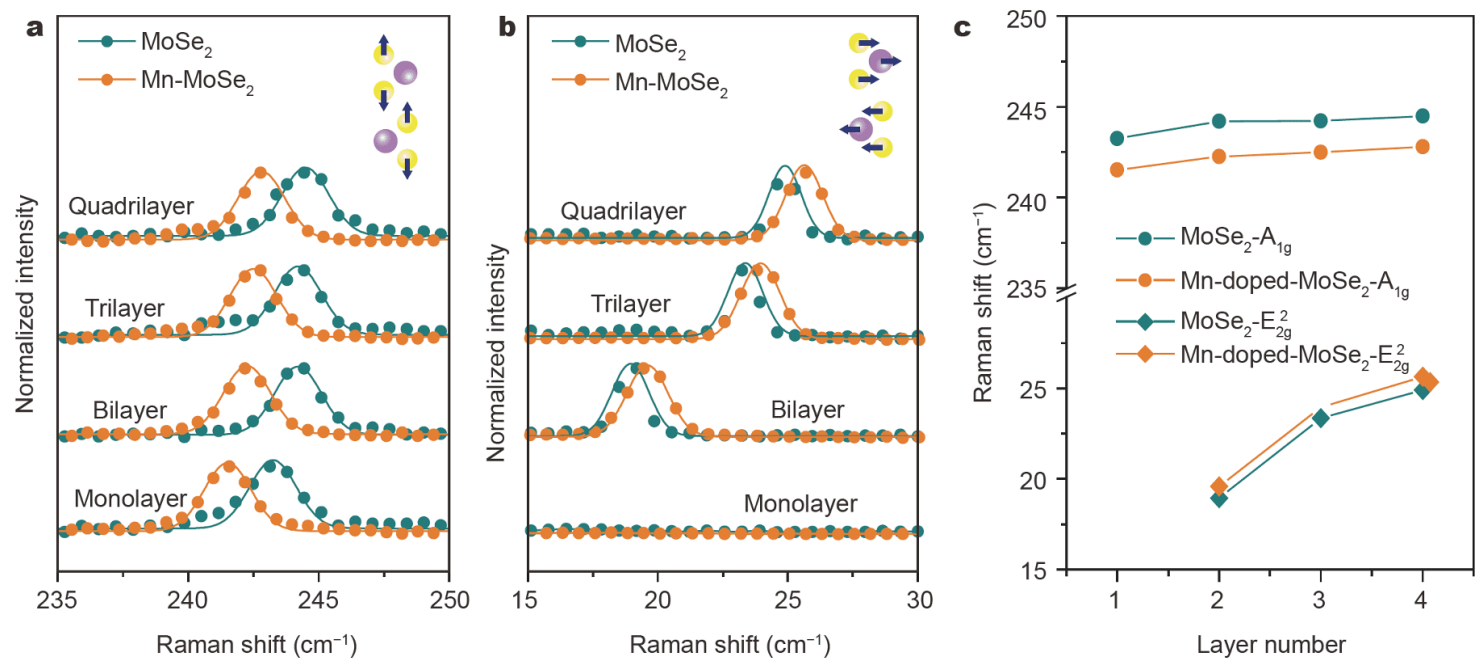

Figure 2 The effect of Mn doping on the vibrational modes of $\mathrm{MoSe}_{2}$ revealed by Raman spectra. (a) Raman spectra in the range of $\mathrm{A}_{1 \mathrm{~g}}$ mode and (b) in the range of the interlayer shear mode $\left(\mathrm{E}_{2 \mathrm{~g}}^{2}\right)$ of the pristine (green) and Mn-doped (orange) $\mathrm{MoSe}_{2}$ from mono- to quadri-layer. The inset cartoons in (a) and (b) illustrate the vibrational modes of $A_{1 g}$ and interlayer shear mode $\left(E_{2 g}^{2}\right)$, respectively. (c) Layer-dependent Raman peak positions of the pristine (green) and Mn-doped (orange) $\mathrm{MoSe}_{2}$. The $\mathrm{A}_{1 \mathrm{~g}}$ mode of Mn-doped $\mathrm{MoSe}_{2}$ (orange circle) shows a red-shift compared with the pristine $\mathrm{MoSe}_{2}$ (green circle), while the shear mode of the Mn-doped $\mathrm{MoSe}_{2}$ (orange diamond) shows a blue-shift compared with pristine MoSe ${ }_{2}$ (green diamond).

which were measured by scanning the excitation beam in a 2D matrix. The insets are the corresponding optical micrographs, indicating the lateral scale and the color contrast between the mono- (light purple) and bilayers (dark purple). The monolayer regions of both samples present strong PL emissions, while the bilayers have nearly no emission. The spectral peak positions of the PL emissions are denoted by the color contrast (see the scale bar besides). The pristine (red) and Mn-doped $\mathrm{MoSe}_{2}$ (blue) monolayers have different PL peak positions. In Fig. 3b, two typical PL spectra subtracted from the mapping are shown. The PL peak linewidth of the Mndoped monolayer is as narrow as that for the pristine sample, which indicates that the Mn-doped monolayer has a high crystalline quality. Temperature-dependent PL (please see below) and systematic analyses in Supplementary information Note 1 confirm that the PL spectrum relates to intrinsic properties of the exciton and trion. The PL peak position of the Mn-doped $\mathrm{MoSe}_{2}$ monolayer blue-shifts $10 \mathrm{~nm}(\sim 20 \mathrm{meV})$ from the pristine one, which is not clearly observed in the previous study using CVD growth of Mn-doped $\mathrm{MoS}_{2}$ due to prominent broadening of PL linewidth. Samples grown by CVD may contain a significant amount of unintended defects that can affect the PL result. More generally, direct interpretation of the differences in PL peak positions of the $\mathrm{MoSe}_{2}$ and Mn-doped $\mathrm{MoSe}_{2}$ samples is hindered by the possibility of different concentrations of unintended defects in the two samples.

TRPL spectra of the two samples (Fig. 3c) provide further evidence of the high quality of the Mn-doped $\mathrm{MoSe}_{2}$ monolayers. The TRPL spectra display only one decay channel, and thus can be fitted with a mono-exponential decay function. The PL lifetime of the Mndoped $\mathrm{MoSe}_{2}$ monolayer is around $5.83 \pm 0.06 \mathrm{~ns}$, which is about twice that of the pristine monolayer $(2.24 \pm 0.02 \mathrm{~ns})$. It is also worthwhile noting that the fluorescence lifetime image of the Mn-doped and pristine monolayers is homogeneous (as shown in Fig. S2), suggesting that the lifetime difference is related to the intrinsic exciton recombination.

To resolve the exciton behaviors, we measured the temperature-dependent PL of the monolayer samples. The PL spectra of the Mn-doped (orange trace in Fig. 3d left panel) and pristine samples (green trace in Fig. 3d right panel) are plotted as stacked lines for different temperatures. The $20-\mathrm{meV}$ difference between the exciton peaks of the pristine and $\mathrm{Mn}$-doped $\mathrm{MoSe}_{2}$ always exists at all the temperatures, and the peak positions and linewidths of the two samples have similar temperature-dependence (see the fitting details in Fig. S3). By carefully examining the PL peak evolution, one can see distinct excitonic features between the Mn-doped and pristine $\mathrm{MoSe}_{2}$ monolayers. The trion peak of the pristine sample 

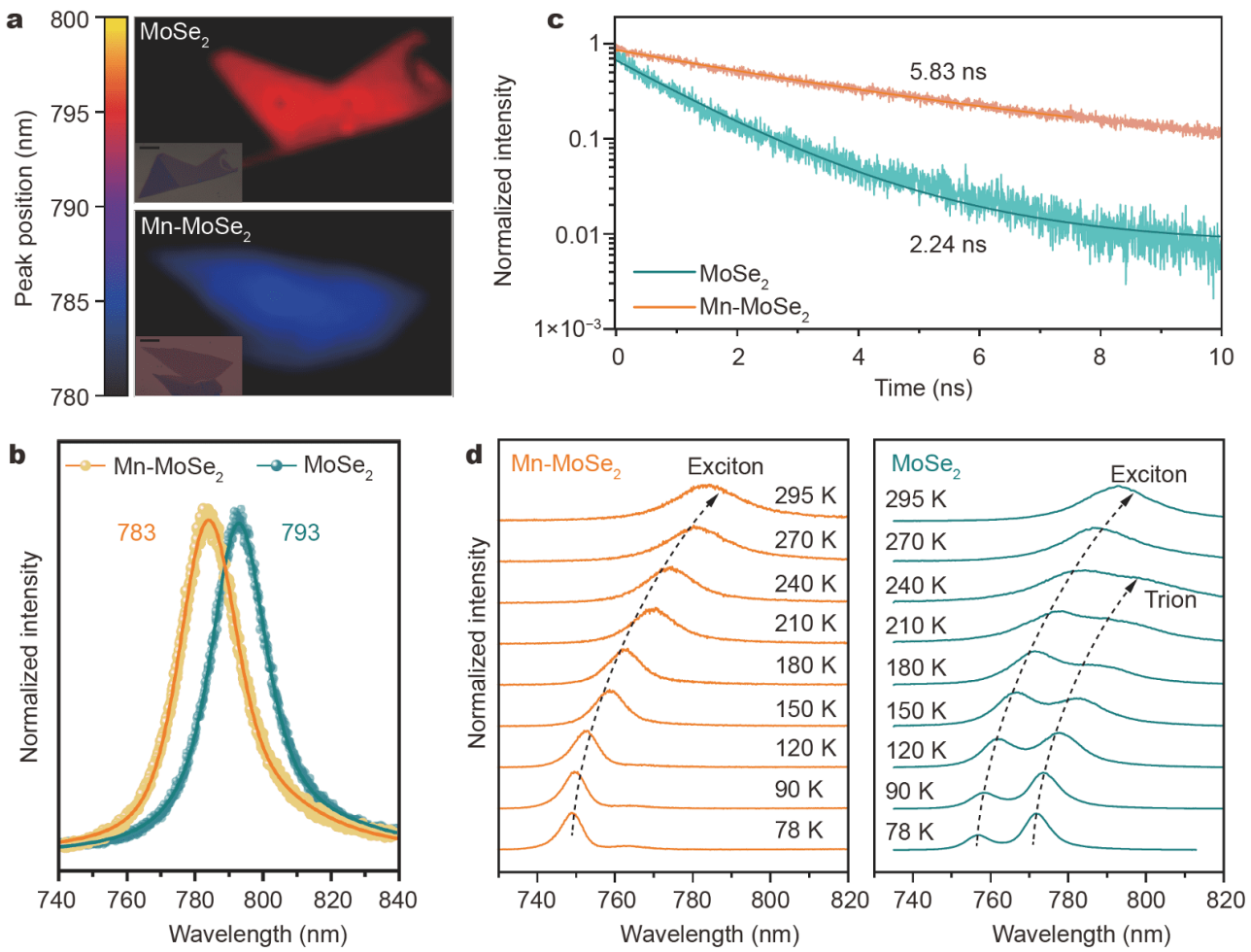

Figure 3 The PL, time-resolved and temperature-dependent PL of the pristine and Mn-doped $\mathrm{MoSe}_{2}$ monolayers. (a) PL peak position mapping of the pristine (red) and Mn-doped (blue) $\mathrm{MoSe}_{2}$ monolayers at room temperature; the insets are optical micrographs of the samples. (b) Typical spectra extracted from the mapping, showing the peak positions. (c) TRPL spectra of the pristine (green) and Mn-doped (orange) MoSe $e_{2} m_{0}$ temperature. (d) Temperature-dependent PL spectra of the pristine (green) and Mn-doped (orange) $\mathrm{MoSe}_{2}$ monolayers down to liquid nitrogen temperature.

is more prominent than the exciton peak at liquid nitrogen temperature, and stays robust until the temperature exceeds $240 \mathrm{~K}$. In stark contrast to the pristine sample, the Mn-doped sample hardly shows any trion emission even at $78 \mathrm{~K}$. From these results, it can be inferred that the Mn-doping can suppress the formation of trions and reduce the trion population in the $\mathrm{Mn}$-doped $\mathrm{MoSe}_{2}$ monolayer. This observation is consistent with the contrasting carrier type and concentrations in devices made from the $\mathrm{MoSe}_{2}$ and $\mathrm{Mn}$-doped $\mathrm{MoSe}_{2}$ monolayers. Specifically, the field-effect transistor (FET) based on the $\mathrm{Mn}$-doped $\mathrm{MoSe}_{2}$ monolayer presents a bipolar performance (see Fig. S4), while the FET based on the pristine $\mathrm{MoSe}_{2}$ behaves as a conventional n-type device with a larger concentration of electron carriers, favoring a larger trion population.

Comparing the PL spectra of the Mn-doped and pristine $\mathrm{MoSe}_{2}$ monolayers, no new emission peaks related to active transition of defect states were detected. However, prolonged PL lifetime and suppressed trion population were observed in the Mn-doped sample, which implies that the band edge excitonic recombination can be af- fected by the magnetic dopants and local dynamics. We measured the circular-polarization-resolved PL of the $\mathrm{Mn}$-doped and pristine $\mathrm{MoSe}_{2}$ monolayers at $10 \mathrm{~K}$ with an applied magnetic field along the out-of-plane direction. Fig. 4a shows the left- $\left(\sigma^{+}\right.$, in red) and right-handed $\left(\sigma^{-}\right.$, in blue) polarized PL spectra at $5 \mathrm{~T}$. For both $\mathrm{Mn}-$ doped and pristine $\mathrm{MoSe}_{2}$, a clear energy offset of both exciton $(\mathrm{X})$ and trion $\left(\mathrm{X}^{-}\right)$peaks can be seen between $\sigma^{+}$ and $\sigma^{-}$spectra. This energy offset has been attributed to the magnetic-field-induced lifting of the degeneracy between the $\mathrm{K}^{+}$and $\mathrm{K}^{-}$valleys [44-47], which is different for the valence and conduction states. We refered to the difference between the PL peak positions for $\sigma^{+}$and $\sigma^{-}$ spectra as the valley Zeeman splitting. Under further examination, we observed that Mn-doped $\mathrm{MoSe}_{2}$ monolayer has more prominent valley Zeeman splitting than the pristine $\mathrm{MoSe}_{2}$ monolayer. Fig. $4 \mathrm{~b}$ plots the dependence of the valley Zeeman splitting on magnetic field for the Mn-doped (orange traces) and pristine (green traces) $\mathrm{MoSe}_{2}$ monolayers. With offsetting the energy position without magnetic field to zero, the results from exciton (solid circle) and trion (open circle) peaks are nearly 

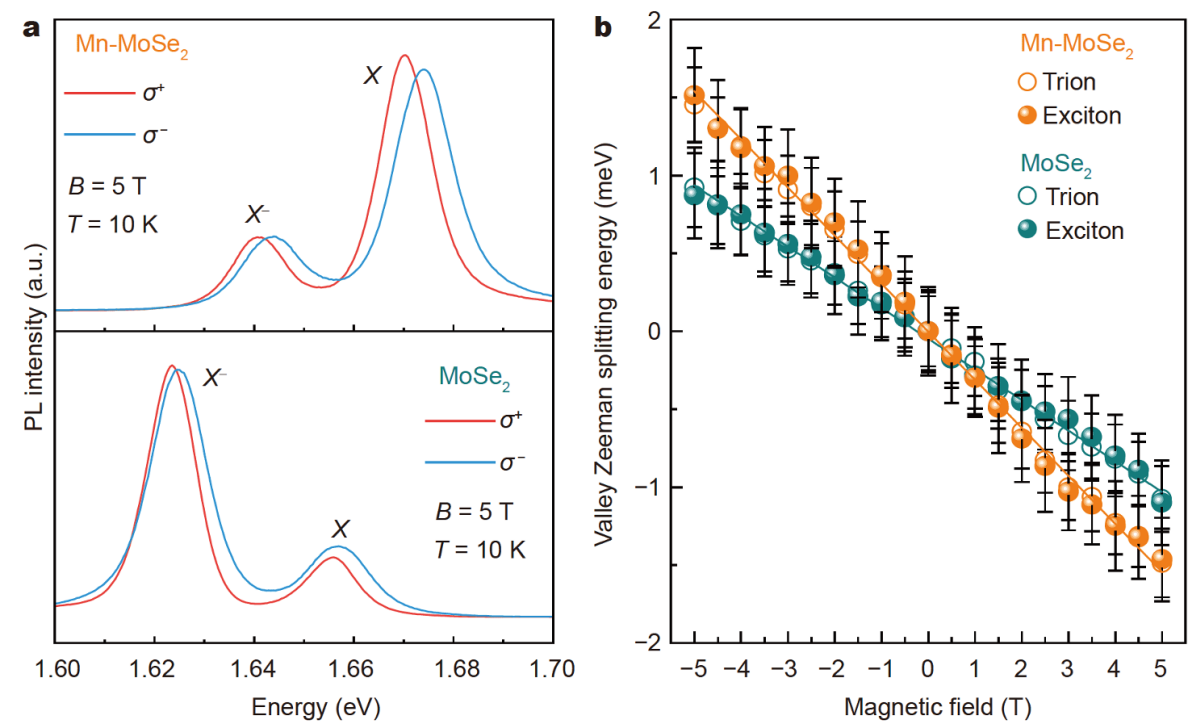

Figure 4 Enhanced valley-Zeeman splitting of the Mn-doped $\mathrm{MoSe}_{2}$ monolayer compared with the pristine MoSe $\mathrm{C}_{2}$ monolayer. (a) Circularpolarization-resolved PL spectra of the Mn-doped (upper panel) and pristine (lower panel) $\mathrm{MoSe}_{2}$ monolayers under magnetic field of $5 \mathrm{~T}$. The leftand right-handed polarized PL are in red and blue, respectively. (b) Dependence of valley-Zeeman splitting on magnetic field of the Mn-doped (orange traces) and pristine (green traces) $\mathrm{MoSe}_{2}$ monolayers. Results of exciton and trion are denoted in solid and open circles, respectively. The solid lines show the linear fits.

identical and present linear dependence to the field. From the linear fits, the slopes for the Mn-doped and pristine $\mathrm{MoSe}_{2}$ monolayers are $-0.302 \pm 0.004$ and $-0.197 \pm$ $0.002 \mathrm{meV} \mathrm{T}^{-1}$, respectively. The valley $g$-factor $\left(g_{\mathrm{v}}\right)$ was defined as [47]:

$g_{\mathrm{v}}=\frac{E_{\sigma^{+}}-E_{\sigma^{-}}}{\mu_{\mathrm{B}} B}$

where $E_{\sigma^{+}}$and $E_{\sigma^{-}}$are the measured exciton (trion) peak energy in $\sigma^{+}$and $\sigma^{-}$spectra, respectively; $\mu_{\mathrm{B}}$ is the Bohr magneton, and $B$ is the magnetic field. The valley $g$-factor of the $\mathrm{Mn}$-doped and pristine $\mathrm{MoSe}_{2}$ monolayers are $-5.22 \pm 0.13$ and $-3.41 \pm 0.07$. The valley Zeeman splitting of the $\mathrm{MoSe}_{2}$ has thus been enhanced by $\sim 50 \%$ due to the Mn dopants.

Due to the spin-valley lock-in, the valley Zeeman splitting in a magnetic field arises from the intrinsic chirality of Bloch electrons at $\mathrm{K}^{+}$and $\mathrm{K}^{-}$points. Previous reports about TMD on ferromagnetic substrate demonstrated that valley Zeeman splitting is greatly enhanced via magnetic-proximity-effect $[48,49]$. However, this enhanced splitting has nonlinear dependence on the magnetic field, which cannot explain the linear dependence that we observed in the $\mathrm{Mn}$-doped $\mathrm{MoSe}_{2}$ monolayer.

To understand the role of $\mathrm{Mn}$ doping on the above observations, spin-polarized DFT calculations $[29,30]$ on the $\mathrm{MoSe}_{2}$ monolayers with $\mathrm{Mn}_{\mathrm{Mo}}$ substitutional defects were performed. In line with previous studies [36,37], the
DFT $+U$ approach [50] was used to account for correlation effects associated with the Mn d orbitals, with $U=$ $3 \mathrm{eV}(U=4 \mathrm{eV}$ gives similar results.) We first computed the Raman modes of the pristine $\mathrm{MoSe}_{2}$ and the Mndoped system, using a $4 \times 4 \times 1$ supercell of $\mathrm{MoSe}_{2}$ with one Mo atom replaced by one $\mathrm{Mn}$ atom $\left(\mathrm{Mn}_{\mathrm{Mo}}\right)$ (see Fig. $5 \mathrm{a}$ ). It was observed that the Mn-Se bond lengths (2.41 $\AA$ ) are smaller than the Mo-Se bond lengths in the pristine $\mathrm{MoSe}_{2}(2.51 \AA)$. At the same time, the distance between $\mathrm{Mn}$ and its nearest Mo neighbors (3.28 $\AA$ ) is larger compared with the Mo-Mo distance in the pristine $\mathrm{MoSe}_{2}(3.25 \AA)$, indicating a weakening of the Mo-Se bonds in the immediate vicinity of $\mathrm{Mn}_{\mathrm{Mo}}$. The computed frequency of the $A_{1 g}$ mode is smaller in the Mn-doped system compared with the pristine system, as shown in Fig. 5b. Although the $\mathrm{Mn}$ concentration in the $4 \times 4$ supercell $(6.25 \%)$ is larger than that estimated in experiment, Fig. $5 \mathrm{~b}$ shows that the calculations and experiment have similar trends in the frequency of the $A_{1 g}$ mode when plotted as a function of $\mathrm{Mn}$ concentration. These results also confirm that the Mn defects in experiment are very likely to be $\mathrm{Mn}_{\mathrm{Mo}}$ [51,52]. The $\mathrm{A}_{1 \mathrm{~g}}$ mode in the pristine $\mathrm{MoSe}_{2}$ is illustrated schematically in Fig. 2a, and our calculations show that the $A_{1 g}$ mode eigenvector of the doped system is about $97 \%$ identical to that of the pristine system. The red-shift of the $\mathrm{A}_{1 \mathrm{~g}}$ mode in the Mndoped system can be explained by the smaller magnitude of the force constants related to the atomic vibrations 
a
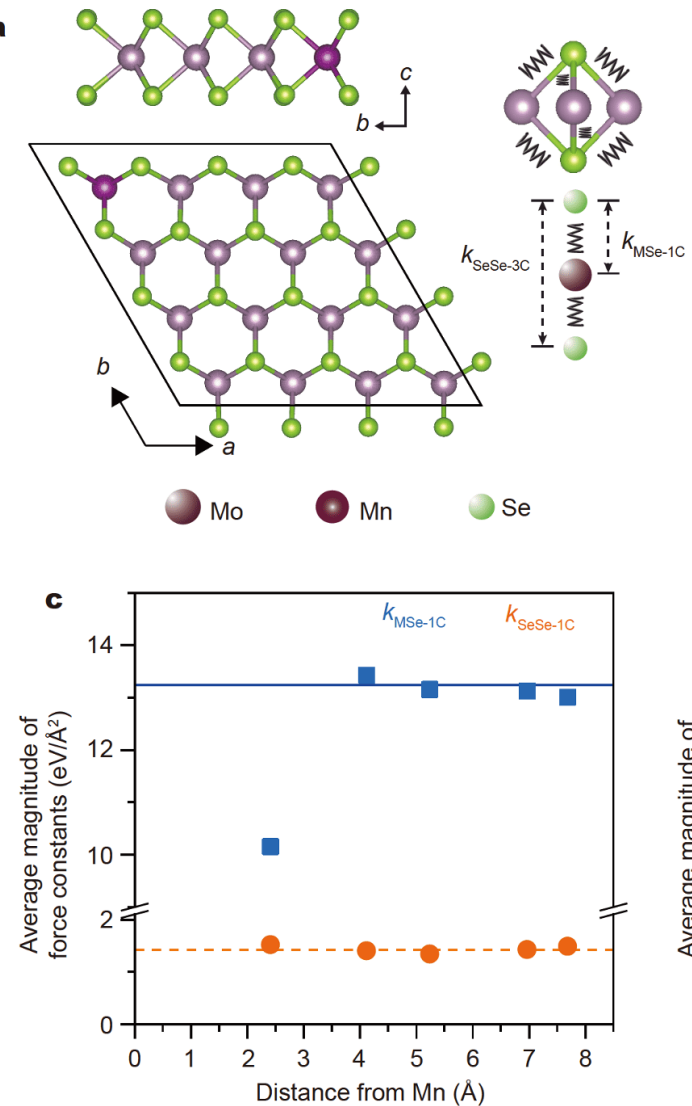
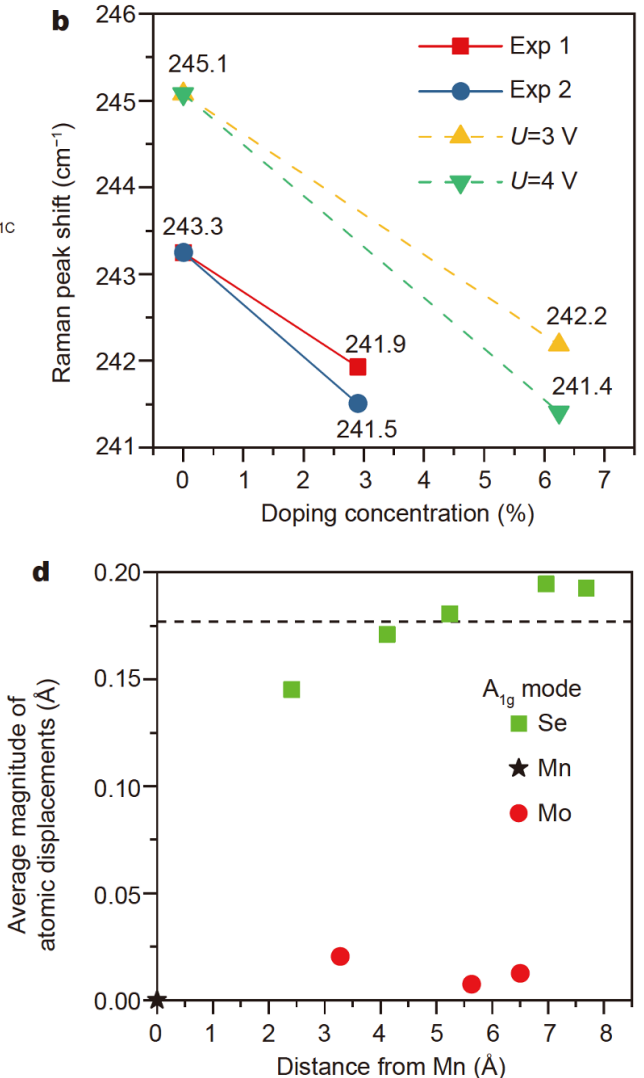

Figure 5 Theoretical analyses of $A_{1 g}$ Raman shift in the Mn-doped $\mathrm{MoSe}_{2}$. (a) Atomic structure of a $4 \times 4 \times 1 \mathrm{MoSe}_{2}$ supercell, with one Mo replaced by $\mathrm{Mn}$ (i.e., $\mathrm{Mn}_{\mathrm{MO}}$ ), viewed along the $2 \mathrm{D}$ plane as well as from top down. An illustration of the $k_{\mathrm{MSe}-1 \mathrm{C}}$ and $k_{\mathrm{SeSe}-3 \mathrm{C}}$ force constants is also included on the right. In this schematic illustration, we simplify the 3D interactions between metal and Se atoms at each Se site into a linear chain along the $z$-direction (b) Raman shift $\left(\mathrm{cm}^{-1}\right)$ of the $\mathrm{A}_{1 \mathrm{~g}}$ mode plotted as a function of Mn (doping) concentration for both theory and experiment. (c) Magnitude of force constants $k_{\mathrm{MSe}-1 \mathrm{C}}$ and $k_{\mathrm{SeSe}-3 \mathrm{C}}$ plotted against distance away from $\mathrm{Mn}_{\mathrm{Mo}}$, averaged over all atoms at the same distance away from $\mathrm{Mn}_{\mathrm{Mo}}$. The solid blue and dashed orange lines represent the magnitudes of $k_{\mathrm{MSe}-1 C}$ and $k_{\mathrm{SeS}-3 C}$ in the pristine $\mathrm{MoSe}_{2} 4 \times 4 \times 1$ supercell, respectively. (d) Average amplitude of atomic displacements $(\AA)$ in the $\mathrm{A}_{\mathrm{lg}}$ mode, plotted against distance away from $\mathrm{Mn}_{\mathrm{Mo}}$. The dotted horizontal line represents the amplitude of atomic displacements $(\AA)$ of Se atoms in a pristine $4 \times 4 \times 1 \mathrm{MoSe}_{2}$ supercell.

[53]. Specifically, our calculations show that the compressive force $(C)$ constant for the nearest neighbor $\mathrm{M}-\mathrm{Se}$ bonds, $k_{\mathrm{MSe}-1 \mathrm{C}}$, is significantly smaller in the immediate vicinity of the Mn dopant site (Fig. 5c), when compared with the corresponding force constants in the pristine $\mathrm{MoSe}_{2}$, computed for the same supercell (horizontal lines in Fig. 5c). This is consistent with the changes in bond lengths close to the Mn dopant as discussed above.

Furthermore, while Mo atoms in the pristine systems are stationary in the $\mathrm{A}_{1 \mathrm{~g}}$ mode, Mo atoms have small but nonnegligible amplitudes of displacements in the Mndoped case (see Fig. 5d), increasing the effective mass associated with the $A_{1 g}$ mode and thereby contributing also to a red-shift of the $A_{1 g}$ frequency.

We also comment that the red-shift of the $A_{1 g}$ mode observed in experiment is unlikely to be due to strain.
Firstly, the samples are mechanically exfoliated from a CVT-grown bulk sample, excluding the effects of the substrate-induced strain. Secondly, our calculations on $\mathrm{MoSe}_{2}$ monolayers with a $\mathrm{Mn}$ doping concentration of $6.25 \%$ show that the Mn dopants induce only minimal strain on the system $(\sim-0.2 \%$ for calculations with $U=$ $3 \mathrm{eV}$ ). Taking into account this small lattice strain does not change the frequency of the $\mathrm{A}_{1 \mathrm{~g}}$ mode.

Thus, the red shift of the $A_{1 g}$ mode arises from the softening of the intralayer force constants due to the weakened Mo-Se bonds close to the Mn dopants. These arguments do not apply to the $\mathrm{E}_{2 \mathrm{~g}}^{2}$ mode because the $\mathrm{E}_{2 \mathrm{~g}}^{2}$ mode is an interlayer mode, which does not involve any relative atomic displacements within the layers. Instead, the blue shift of the $E_{2 \mathrm{~g}}^{2}$ mode is consistent with the reduced average mass per unit area of the doped layers, as 
Mn has a smaller atomic mass than Mo. For the interlayer shear mode $\mathrm{E}_{2 \mathrm{~g}}^{2}$, Zhao et al. [54] showed that its phonon frequency has the relationship $\omega^{2}\left(\mathrm{E}_{2 \mathrm{~g}}^{2}\right) \propto K / m$, where $K$ is the nearest neighbor inter-trilayer shear force constant and $m$ is the average mass per unit area of the monolayer. At the experimental doping level of $2.9 \%$, the average mass of the monolayer decreases by $0.47 \%$, which would result in a blue shift of $0.04 \mathrm{~cm}^{-1}$ if the force constant $K$ remains the same. The larger blue-shift observed in experiment suggests that the interlayer force constant $K$ increases by about $7 \%$, which may be due to the increased Coulombic interactions between the doped layers.

We studied the effects of $\mathrm{Mn}_{\mathrm{Mo}}$ on the electronic and magnetic properties of the $\mathrm{MoSe}_{2}$ by using a larger $6 \times 6 \times 1$ supercell of $\mathrm{MoSe}_{2}$, corresponding to a uniformly doped $\mathrm{MoSe}_{2}$ monolayer with a doping concentration of $2.78 \%$, close to the experimental doping level of $2.9 \%$. The Mn dopants result in spin-polarized in-gap states, as shown in the band structure in Fig. 6a. The energy differences between these states and the valence and conduction band edges are significantly larger than thermal energy at room temperature, in agreement with previous reports [36,37]. The in-gap states comprise five separate bands, with one band below the Fermi level, a pair of energy-degenerate bands just above the Fermi level and two bands about $0.5 \mathrm{eV}$ above the Fermi level. From the projected densities of states (see Fig. S5), we see that these in-gap states arise from the Mn 3d orbitals, as well as from the $4 \mathrm{p}$ orbitals of the nearest-neighbor Se atoms, and the $4 \mathrm{~d}$ orbitals of the next nearest neighbor Mo atoms. Contributions from other atoms are negligible. The projected densities of states (PDOS) on individual orbitals from these relevant atoms are shown in Fig. S6. In Fig. 6a, the in-gap states are labelled according to the Mn $3 \mathrm{~d}$ character $\left(\mathrm{d}_{z^{2}} ; \mathrm{d}_{x y}\right.$ and $\mathrm{d}_{x^{2}-y^{2}} ; \mathrm{d}_{x z}$ and $\mathrm{d}_{y z}$ ) of these states. Crystal field splitting is observed according to the degree of overlap of these $d$ orbitals with the nearest neighbor Se atoms, as described in [36]. The magnetic moment on Mn atoms $\left(2.38 \mu_{\mathrm{B}}\right)$ is about twice the total net magnetic moment in the supercell $\left(1.30 \mu_{B}\right)$. As shown in Fig. $6 \mathrm{~b}$, the Se and Mo atoms closest to $\mathrm{Mn}$ carry small magnetic moments which are opposite in sign to that on Mn (Se: $-0.08 \mu_{B}$, Mo: $\left.-0.07 \mu_{\mathrm{B}}\right)$.

The deep in-gap states observed for the Mn-doped $\mathrm{MoSe}_{2}$ suggest the presence of a local defect potential [55]. In order to assess the effectiveness of this local potential in trapping excitons, we plotted the local valence band maximum (VBM) and conduction band minimum (CBM) as a function of distance from Mn (Fig. 6c). These local VBM and CBM were defined as the band edges in the PDOS on Mo atoms. The local VBM shifts up and the local CBM shifts down as the distance between Mo and
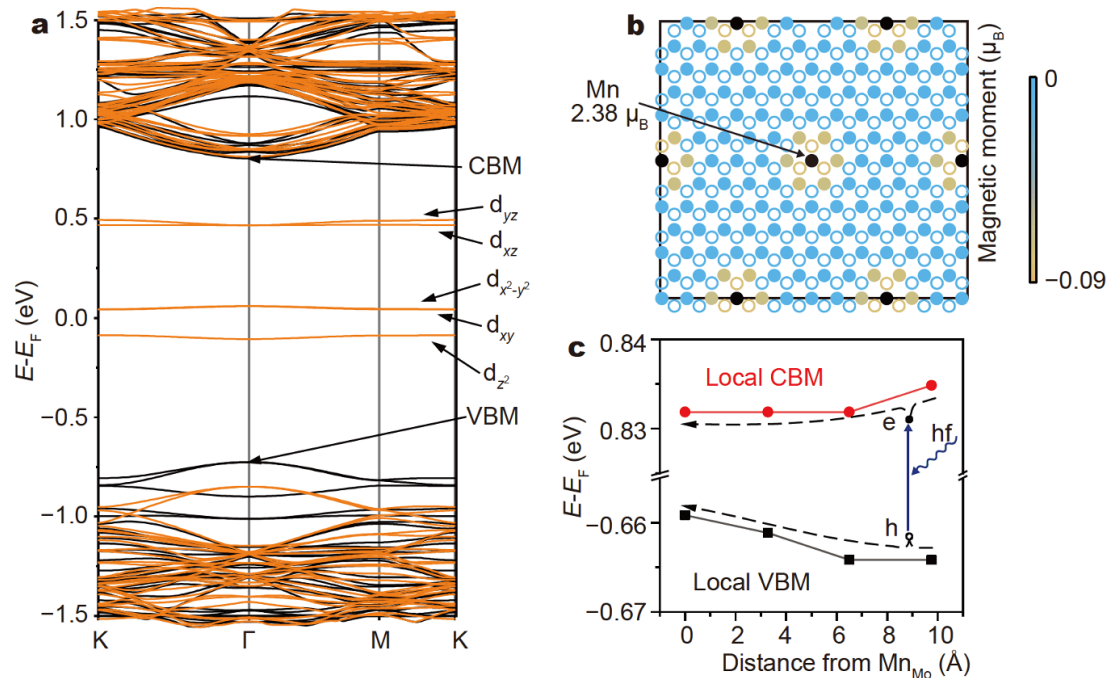

Figure 6 Electronic structure of the Mn-doped $\mathrm{MoSe}_{2}$, obtained with a $6 \times 6 \times 1$ supercell. (a) Spin-polarized band structure of the Mn-doped MoSe $\mathrm{e}_{2}$. The VBM and CBM are labelled, together with the character of the defect in-gap states. Orange denotes spin up and black denotes spin down. (b) Schematic heat map showing magnetic moments of the atoms. Mn (solid black circles) atoms have a magnetic moment of $2.38 \mu_{\mathrm{B}}$, and the Mo (solid circles) and Se (hollow circles) closest to $\mathrm{Mn}$ show antiferromagnetic alignment to $\mathrm{Mn}$, while other atoms have negligible magnetic moments. (c) Local VBM and CBM as a function of distance from Mn. The local VBM and CBM are defined respectively as the frontier valence and conduction band edges in the projected densities of states on Mo atoms at a given distance from Mn. Dashed arrows illustrate the funnelling of an exciton toward the $\mathrm{Mn}_{\mathrm{Mo}}$ defect site. 
Mn decreases, suggesting the presence of a Type I funnel [56] for the excitons toward the Mn site. This analysis holds for $\mathrm{Mn}_{\mathrm{Mo}}$ dopants that are fairly uniformly distributed. In this case, the exciton radius [57] is also expected to be large enough to cover some Mn sites. In another scenario where the $\mathrm{Mn}$ dopants are clustered, and the exciton is created in a region of $\mathrm{MoSe}_{2}$ with very few dopants, we also predict that the VBM and CBM alignment favor the funnelling of the excitons towards the more highly doped region for the large exciton binding energies expected here (see Fig. S7). Our results therefore suggest that the excitons are likely to be funnelled toward the Mn defect sites. The trapping of excitons can contribute to a reduction in the density of trions $[58,59]$ as observed in the Mn-doped samples (Fig 3c). The funnelling of excitons to Mn sites also leads to large exchange interactions [46] between the excitons and the Mn defects, consistent with the large valley splitting observed in Fig. 4. With increasing magnetic field, we expect the total net spin of the Mn sites in the experimental sample to increase. In the case where all the spins are ferromagnetically aligned, our calculations with a $4 \times 4 \times 1$ supercell including the effects of spin-orbit coupling reveal that the breaking of time-reversal symmetry caused by the aligned Mn magnetic moments induces a significant lifting of the energy degeneracy between the VBM at $\mathrm{K}^{+}$and the VBM at $\mathrm{K}^{-}$, of $\sim 0.16 \mathrm{eV}$ (see Fig. S8a). The VBM wavefunctions in this system have a larger weight close to the Mn sites, consistent with the discussion on Fig. $6 c$ for the $6 \times 6 \times 1$ supercell. The linear dependence of the valley Zeeman splitting on the external magnetic field shown in Fig. $4 \mathrm{~b}$ suggests that the net magnetic moment in Mn-doped $\mathrm{MoSe}_{2}$ increases linearly with the magnetic field strength [46].

Mn has one more valence electron than Mo, which suggests that the enhanced valley Zeeman splitting could be due to the magnetic response from free electrons [60]. However, our Bader charge calculations indicate that compared with $\mathrm{Mo}, \mathrm{Mn}$ in fact donates less electrons to surrounding anions (see Fig. S9). These observations are consistent with the reduction in trion population and the bipolar character of the Mn-doped $\mathrm{MoSe}_{2}$ device. We also note that the $\mathrm{Mn}_{\mathrm{Mo}}$ are most stable as neutral defects (see Fig. S10), and it takes a large amount of energy $(>1.43 \mathrm{eV})$ to transfer an electron from the $\mathrm{Mn}_{\mathrm{Mo}}$ defect states to the conduction band.

\section{CONCLUSIONS}

In summary, we synthesized the Mn-doped $\mathrm{MoSe}_{2}$ single crystal by CVT reactions with $\mathrm{AlCl}_{3}$ as a transport agent.
XRD, STEM and EDX results demonstrate high crystal quality with a $\mathrm{Mn}$ atomic concentration up to $2.9 \%$. The $\mathrm{Mn}$ doping causes the interlayer $\mathrm{E}_{2 \mathrm{~g}}^{2}$ and intralayer $\mathrm{A}_{\mathrm{lg}}$ Raman modes to blue-shift and red-shift, respectively. First principles calculations on $\mathrm{Mn}_{\mathrm{Mo}}$ defects in $\mathrm{MoSe}_{2}$ monolayers also predict a red-shift in the $A_{1 g}$ mode, lending evidence to the nature of the Mn defects in experiment. The red-shift of the $A_{1 g}$ mode can be attributed to a weakening of the intralayer compressive force constants as well as the atomic displacements of Mo atoms in the $\mathrm{A}_{\mathrm{lg}}$ mode. The exfoliated monolayer from the Mndoped $\mathrm{MoSe}_{2}$ shows a suppressed trion peak and a longer PL lifetime compared with the pristine counterpart. Moreover, its valley Zeeman splitting is enhanced by $\mathrm{Mn}$ doping, such that the $g$-factor increases by $\sim 50 \%$. Our first principles calculations predict that Mn dopants carry a large magnetic moment of $\sim 2.38 \mu_{\mathrm{B}}$. The calculations also suggest that these magnetic dopants result in the funnelling of $\mathrm{MoSe}_{2}$ excitons toward the $\mathrm{Mn}$ atoms, consistent with the suppressed trion peak and the increase in valley Zeeman splitting. Although ferromagnetism is not realized in our CVT grown Mn-doped $\mathrm{MoSe}_{2}$, the local magnetic moment significantly enhances the exciton $g$-factors via exchange interaction, highlighting the potential application of the Mn-doped $\mathrm{MoSe}_{2}$ monolayers as future spintronic and valleytronic materials.

Received 11 December 2020; accepted 7 February 2021; published online 8 May 2021

1 Splendiani A, Sun L, Zhang Y, et al. Emerging photoluminescence in monolayer $\mathrm{MoS}_{2}$. Nano Lett, 2010, 10: 1271-1275

2 Mak KF, Lee C, Hone J, et al. Atomically thin $\mathrm{MoS}_{2}$ : A new directgap semiconductor. Phys Rev Lett, 2010, 105: 136805

3 Chernikov A, Berkelbach TC, Hill HM, et al. Exciton binding energy and nonhydrogenic Rydberg series in monolayer $\mathrm{WS}_{2}$. Phys Rev Lett, 2014, 113: 076802

4 Ugeda MM, Bradley AJ, Shi SF, et al. Giant bandgap renormalization and excitonic effects in a monolayer transition metal dichalcogenide semiconductor. Nat Mater, 2014, 13: 1091-1095

5 He K, Kumar N, Zhao L, et al. Tightly bound excitons in monolayer $\mathrm{WSe}_{2}$. Phys Rev Lett, 2014, 113: 026803

6 Granados Del Águila A, Liu S, Do TTH, et al. Linearly polarized luminescence of atomically thin $\mathrm{MoS}_{2}$ semiconductor nanocrystals. ACS Nano, 2019, 13: 13006-13014

7 Mak KF, He K, Shan J, et al. Control of valley polarization in monolayer $\mathrm{MoS}_{2}$ by optical helicity. Nat Nanotech, 2012, 7: 494498

8 Zeng H, Dai J, Yao W, et al. Valley polarization in $\mathrm{MoS}_{2}$ monolayers by optical pumping. Nat Nanotech, 2012, 7: 490-493

9 Behnia K. Polarized light boosts valleytronics. Nat Nanotech, 2012, 7: 488-489

10 Liu S, Granados Del Águila A, Liu X, et al. Room-temperature 
valley polarization in atomically thin semiconductors via chalcogenide alloying. ACS Nano, 2020, 14: 9873-9883

11 Mak KF, McGill KL, Park J, et al. The valley Hall effect in $\mathrm{MoS}_{2}$ transistors. Science, 2014, 344: 1489-1492

12 Lee J, Mak KF, Shan J. Electrical control of the valley Hall effect in bilayer $\mathrm{MoS}_{2}$ transistors. Nat Nanotech, 2016, 11: 421-425

13 Ly TH, Chiu MH, Li MY, et al. Observing grain boundaries in CVD-grown monolayer transition metal dichalcogenides. ACS Nano, 2014, 8: 11401-11408

14 Jeong HY, Lee SY, Ly TH, et al. Visualizing point defects in transition-metal dichalcogenides using optical microscopy. ACS Nano, 2016, 10: 770-777

15 Lee Y, Yun SJ, Kim Y, et al. Near-field spectral mapping of individual exciton complexes of monolayer $\mathrm{WS}_{2}$ correlated with local defects and charge population. Nanoscale, 2017, 9: 2272-2278

$16 \mathrm{Lu}$ J, Carvalho A, Chan XK, et al. Atomic healing of defects in transition metal dichalcogenides. Nano Lett, 2015, 15: 3524-3532

17 Lin YC, Dumcenco DO, Komsa HP, et al. Properties of individual dopant atoms in single-layer $\mathrm{MoS}_{2}$ : Atomic structure, migration, and enhanced reactivity. Adv Mater, 2014, 26: 2857-2861

18 Cadiz F, Courtade E, Robert C, et al. Excitonic linewidth approaching the homogeneous limit in $\mathrm{MoS}_{2}$-based van der Waals heterostructures. Phys Rev X, 2017, 7: 021026

19 Wang X, Du K, Liu YYF, et al. Raman spectroscopy of atomically thin two-dimensional magnetic iron phosphorus trisulfide $\left(\mathrm{FePS}_{3}\right)$ crystals. 2D Mater, 2016, 3: 031009

20 Du K, Wang X, Liu Y, et al. Weak van der Waals stacking, widerange band gap, and raman study on ultrathin layers of metal phosphorus trichalcogenides. ACS Nano, 2016, 10: 1738-1743

21 Li H, Duan X, Wu X, et al. Growth of alloy $\mathrm{MoS}_{2 x} \mathrm{Se}_{2(1-x)}$ nanosheets with fully tunable chemical compositions and optical properties. J Am Chem Soc, 2014, 136: 3756-3759

22 Feng Q, Zhu Y, Hong J, et al. Growth of large-area 2D $\mathrm{MoS}_{2(1-x)} \mathrm{Se}_{2}$ ${ }_{x}$ semiconductor alloys. Adv Mater, 2014, 26: 2648-2653

23 Komsa HP, Krasheninnikov AV. Two-dimensional transition metal dichalcogenide alloys: Stability and electronic properties. J Phys Chem Lett, 2012, 3: 3652-3656

24 Chen Y, Xi J, Dumcenco DO, et al. Tunable band gap photoluminescence from atomically thin transition-metal dichalcogenide alloys. ACS Nano, 2013, 7: 4610-4616

25 Zhang K, Feng S, Wang J, et al. Manganese doping of monolayer $\mathrm{MoS}_{2}$ : The substrate is critical. Nano Lett, 2015, 15: 6586-6591

26 Chua XJ, Luxa J, Eng AYS, et al. Negative electrocatalytic effects of p-doping niobium and tantalum on $\mathrm{MoS}_{2}$ and $\mathrm{WS}_{2}$ for the hydrogen evolution reaction and oxygen reduction reaction. ACS Catal, 2016, 6: 5724-5734

27 Das S, Demarteau M, Roelofs A. Nb-doped single crystalline $\mathrm{MoS}_{2}$ field effect transistor. Appl Phys Lett, 2015, 106: 173506

28 Suh J, Park TE, Lin DY, et al. Doping against the native propensity of $\mathrm{MoS}_{2}$ : Degenerate hole doping by cation substitution. Nano Lett, 2014, 14: 6976-6982

29 Kresse G, Furthmüller J. Efficient iterative schemes for $a b$ initio total-energy calculations using a plane-wave basis set. Phys Rev B, 1996, 54: 11169-11186

30 Kresse G, Joubert D. From ultrasoft pseudopotentials to the projector augmented-wave method. Phys Rev B, 1999, 59: 1758-1775

31 Blöchl PE. Projector augmented-wave method. Phys Rev B, 1994, 50: $17953-17979$

32 Perdew JP, Zunger A. Self-interaction correction to density-functional approximations for many-electron systems. Phys Rev B,
1981, 23: 5048-5079

33 Rohrbach A, Hafner J, Kresse G. Electronic correlation effects in transition-metal sulfides. J Phys-Condens Matter, 2003, 15: 979996

$34 \mathrm{Wu} \mathrm{M}$, Yao X, Hao $\mathrm{Y}$, et al. Electronic structures, magnetic properties and band alignments of $3 \mathrm{~d}$ transition metal atoms doped monolayer $\mathrm{MoS}_{2}$. Phys Lett A, 2018, 382: 111-115

35 Andriotis AN, Menon M. Tunable magnetic properties of transition metal doped $\mathrm{MoS}_{2}$. Phys Rev B, 2014, 90: 125304

36 Mishra R, Zhou W, Pennycook SJ, et al. Long-range ferromagnetic ordering in manganese-doped two-dimensional dichalcogenides. Phys Rev B, 2013, 88: 144409

37 Ramasubramaniam A, Naveh D. Mn-doped monolayer $\mathrm{MoS}_{2}$ : An atomically thin dilute magnetic semiconductor. Phys Rev B, 2013, 87: 195201

38 Monkhorst HJ, Pack JD. Special points for Brillouin-zone integrations. Phys Rev B, 1976, 13: 5188-5192

39 Togo A, Tanaka I. First principles phonon calculations in materials science. Scripta Mater, 2015, 108: 1-5

40 Tang W, Sanville E, Henkelman G. A grid-based Bader analysis algorithm without lattice bias. J Phys-Condens Matter, 2009, 21: 084204

41 Naik MH, Jain M. CoFFEE: Corrections For Formation Energy and Eigenvalues for charged defect simulations. Comput Phys Commun, 2018, 226: 114-126

42 Döll G, Lux-Steiner MC, Kloc C, et al. Chemical vapour transport and structural characterization of layered $\mathrm{MnIn}_{2} \mathrm{Se}_{4}$ single crystals. J Cryst Growth, 1990, 104: 593-600

43 Gao J, Kim YD, Liang L, et al. Transition-metal substitution doping in synthetic atomically thin semiconductors. Adv Mater, 2016, 28: 9735-9743

44 Jiang C, Liu F, Cuadra J, et al. Zeeman splitting via spin-valleylayer coupling in bilayer $\mathrm{MoTe}_{2}$. Nat Commun, 2017, 8: 802

45 Srivastava A, Sidler M, Allain AV, et al. Valley Zeeman effect in elementary optical excitations of monolayer $\mathrm{WSe}_{2}$. Nat Phys, 2015, 11: $141-147$

46 Li Q, Zhao X, Deng L, et al. Enhanced valley Zeeman splitting in Fe-doped monolayer $\mathrm{MoS}_{2}$. ACS Nano, 2020, 14: 4636-4645

47 MacNeill D, Heikes C, Mak KF, et al. Breaking of valley degeneracy by magnetic field in monolayer $\mathrm{MoSe}_{2}$. Phys Rev Lett, 2015, 114: 037401

48 Zhao C, Norden T, Zhang P, et al. Enhanced valley splitting in monolayer $\mathrm{WSe}_{2}$ due to magnetic exchange field. Nat Nanotech, 2017, 12: 757-762

49 Norden T, Zhao C, Zhang P, et al. Giant valley splitting in monolayer $\mathrm{WS}_{2}$ by magnetic proximity effect. Nat Commun, 2019, 10: 4163

50 Himmetoglu B, Floris A, de Gironcoli S, et al. Hubbard-corrected DFT energy functionals: The $\mathrm{LDA}+\mathrm{U}$ description of correlated systems. Int J Quantum Chem, 2014, 114: 14-49

51 Luo X, Zhao Y, Zhang J, et al. Anomalous frequency trends in $\mathrm{MoS}_{2}$ thin films attributed to surface effects. Phys Rev B, 2013, 88: 075320

52 Lu X, Utama MIB, Lin J, et al. Rapid and nondestructive identification of polytypism and stacking sequences in few-layer molybdenum diselenide by Raman spectroscopy. Adv Mater, 2015, 27: 4502-4508

53 Chow WL, Luo X, Quek SQ, et al. Evolution of Raman scattering and electronic structure of ultrathin molybdenum disulfide by oxygen chemisorption. Adv Electron Mater, 2015, 1: 1400037 
54 Zhao $\mathrm{Y}$, Luo X, Li H, et al. Interlayer breathing and shear modes in few-trilayer $\mathrm{MoS}_{2}$ and WSe 2 . Nano Lett, 2013, 13: 1007-1015

55 Pantelides ST. Deep Centers in Semiconductors: a State-of-the-Art Approach. 2nd ed. Yverdon: Gordon and Breach Science Publishers, 1992

56 Feng J, Qian X, Huang CW, et al. Strain-engineered artificial atom as a broad-spectrum solar energy funnel. Nat Photon, 2012, 6: 866872

57 Qiu DY, da Jornada FH, Louie SG. Optical spectrum of $\mathrm{MoS}_{2}$ : Many-body effects and diversity of exciton states. Phys Rev Lett, 2013, 111: 216805

58 Singh A, Moody G, Tran K, et al. Trion formation dynamics in monolayer transition metal dichalcogenides. Phys Rev B, 2016, 93: 041401

59 Godde T, Schmidt D, Schmutzler J, et al. Exciton and trion dynamics in atomically thin $\mathrm{MoSe}_{2}$ and WSe $\mathrm{W}_{2}$ Effect of localization. Phys Rev B, 2016, 94: 165301

60 Wang Z, Mak KF, Shan J. Strongly interaction-enhanced valley magnetic response in monolayer $\mathrm{WSe}_{2}$. Phys Rev Lett, 2018, 120: 066402

Acknowledgements Xiong Q acknowledges the support from Singapore Ministry of Education via AcRF Tier3 Programme "Geometrical Quantum Materials" (MOE2018-T3-1-002), AcRF Tier2 grant (MOE2017-T2-1-040) and Tier1 grant (RG 194/17). Quek SY acknowledges the funding from the National Research Foundation, Prime Ministers Office, Singapore, under its Medium-Sized Centre Programme. Wu Y acknowledges computational support from Miguel dias Costa. Xuan F acknowledges the funding from MOE2017-T2-2-139. The calculations were performed on the clusters of the CA2DM and the National Supercomputing Centre (NSCC) Singapore.

Author contributions Liu S and Xiong Q conceived the idea and designed the experiments. Liu S and Chaturvedi A synthesized the Mndoped $\mathrm{MoSe}_{2}$ single crystal. Liu S prepared the atomically thin samples and performed the spectroscopic experiments. Wu Y performed the first principles calculations and analysis under the supervision of Quek SY. Liu X fabricated the FET devices and measured the transport properties. Liu S, Wu Y, Granados del Aguila A and Liu X analyzed the results. Liu S, Wu Y, Quek SY and Xiong Q wrote the manuscript. All authors discussed and commented on the manuscript. Quek SY and Xiong Q supervised this collaboration project.

Conflict of interest The authors declare no competing interest.

Supplementary information Experimental details and supporting data are available in the online version of the paper.

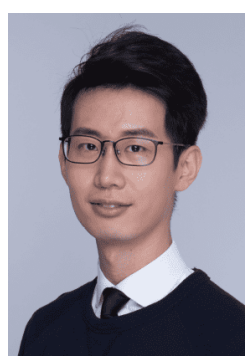

Yaze $\mathbf{W u}$ received his $\mathrm{BSc}$ degree from the Physics Department of National University of Singapore and is currently a $\mathrm{PhD}$ candidate at the same department. His current research interest includes high-throughput first-principles calculations of piezoelectricity and optical properties in $2 \mathrm{D}$ materials.

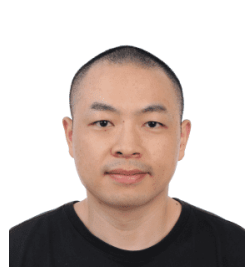

Sheng Liu received his BSc and MSc degrees from the University of Science and Technology Beijing, and $\mathrm{PhD}$ degree from Nanyang Technological University (NTU), Singapore. He is currently a postdoctoral research fellow at Division of Physics and Applied Physics of NTU. His current research interest includes optical properties in $2 \mathrm{D}$ semiconductors and magnets.

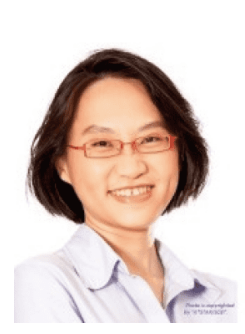

Su Ying Quek is an Associate Professor at the Physics Department, National University of Singapore. She received a $\mathrm{PhD}$ in applied physics from Harvard University and a BA Honors $\left(1^{\text {st }}\right.$ class) in Mathematics from the University of Cambridge. She leads a computation and theory group that studies the electronic structure and lattice dynamics of emerging materials. The group is active in advancing methodologies that address problems of experimental relevance and has strong collaborations with experimentalists.

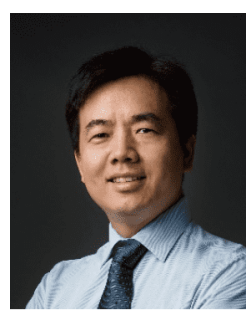

Qihua Xiong is a Professor of physics at Tsinghua University. He received his $\mathrm{PhD}$ from Penn State University in 2006. In 2009-2020 he worked at NTU. His group is specialized in optical spectroscopy investigations of light-matter interactions in low-dimensional quantum materials. He currently serves as Associate Editor for Nano Letters and international advisory board for many prestigious journals, such as ACS Photonics, Nano Research, Science China Materials, etc.

\section{高品质锰掺杂二维二硒化钼晶体的光与物质相互 作用}

刘盛 ${ }^{1 \dagger}$, 武亚则 ${ }^{2,6 \dagger}$, 刘学 ${ }^{1}$, Andres Granados del Aguila ${ }^{1}$, 铉丰源 ${ }^{2}$, Apoorva Chaturvedi ${ }^{3}$, 张华 ${ }^{4,5}$, 郭淑䒨 ${ }^{2,6 *}$, 熊启华 ${ }^{1,7 *}$

摘要 最近, 将磁性掺杂引入到二维硫族化合物中从而调控自旋电 子学及谷电子学的研究引起了科学界的广泛关注. 本文通过化学 气相转移生长技术, 实现了二硒化钼高达 $2.9 \%$ 原子丰度的锰掺杂, 并采用光谱学技术研究了晶体及解理的少层样品中光与物质相互 作用. 我们发现掺杂抑制了带电激子的发光, 激子发光具有更长的 时间寿命, 同时面内 $\mathrm{E}_{2 \mathrm{~g}}{ }^{2}$ 和面外 $\mathrm{A}_{1 \mathrm{~g}}$ 声子振动模式分别呈现出显著的 蓝移和红移. 此外, 锰掺杂增强了能谷塞曼譬裂约 $50 \%$, 并保持了发 光线偏振度对磁场的依赖关系. 第一性原理计算显示, 锰掺杂替代 原子形成自旋极化的深能级, 导致缺陷势场更倾向于捕获激子. 锰 掺杂降低了原子间的相互作用力常数, 可以解释面外 $\mathrm{A}_{1 \mathrm{~g}}$ 声子振动 模式的红移. 锰原子及最近邻的钼和硒原子带有显著的磁偶极, 其 交换作用影响了缺陷捕获的激子, 从而增强了实验中观察到的 $g$-因 子. 